\title{
Cionin, A Vertebrate Cholecystokinin/Gastrin Homolog, Induces Ovulation in the Ascidian Ciona Intestinalis Type A.
}

\section{Tomohiro Osugi}

Suntory Foundation for Life Sciences

Natsuko Miyasaka

Suntory Foundation for Life Sciences

Akira Shiraishi

Suntory Foundation for Life Sciences

\section{Shin Matsubara}

Suntory Foundation for Life Sciences

Honoo Satake ( $\nabla$ satake@sunbor.or.jp )

Suntory Foundation for Life Sciences

\section{Research Article}

Keywords: Cionin, cholecystokinin, gastrin, ovary, ovulation, Ciona robusta, Ciona intestinalis type A, neuropeptide

Posted Date: February 26th, 2021

DOI: https://doi.org/10.21203/rs.3.rs-243411/v1

License: (c) (i) This work is licensed under a Creative Commons Attribution 4.0 International License. Read Full License 


\section{Abstract}

Cionin is a homolog of vertebrate cholecystokinin/gastrin that has been identified in the ascidian Ciona intestinalis type A. The phylogenetic position of ascidians as the closest living relatives of vertebrates suggests that cionin can provide clues to the evolution of endocrine/neuroendocrine systems throughout chordates. Here, we show the biological role of cionin in the regulation of ovulation. In situ hybridization demonstrated that the mRNA of the cionin receptor, Cior2, was expressed specifically in the inner follicular cells of pre-ovulatory follicles in the Ciona ovary. Cionin was found to significantly stimulate ovulation after 24-h incubation. Transcriptome and subsequent Real-time PCR analyses confirmed that the expression levels of receptor tyrosine kinase (RTK) signaling genes and a matrix metalloproteinase (MMP) gene were significantly elevated in the cionin-treated follicles. Of particular interest is that an RTK inhibitor and MMP inhibitor markedly suppressed the stimulatory effect of cionin on ovulation. Furthermore, inhibition of RTK signaling reduced the MMP gene expression in the cionin-treated follicles. These results provide evidence that cionin induces ovulation by stimulating MMP gene expression via the RTK signaling pathway. This is the first report on the endogenous roles of cionin and the induction of ovulation by cholecystokinin/gastrin family peptides in an organism.

\section{Introduction}

Ascidians are the closest living relatives of vertebrates in the Chordata superphylum, and thus they provide important insights into the evolution of peptidergic systems in chordates. Over the past two decades, a wide variety of neuropeptides and their receptors have been identified in the neural complex of Ciona intestinalis Type A (synonym for Ciona robusta) ${ }^{1}$ including cionin, gonadotropin-releasing hormones (GnRHs), tachykinin (CiTK), calcitonin, vasopressin (CiVP), and insulin-like peptide ${ }^{2-10}$. Peptidomic analyses of the neural complex of Ciona have also identified more than 30 neuropeptides and peptide hormones, including various Ciona-specific peptides as well as homologous peptides ${ }^{11,12}$. Furthermore, our recent machine learning-based approach elucidated receptors for galanin-like peptide and 11 novel (Ciona-specific) neuropeptides ${ }^{13}$. In contrast, sexual steroidogenesis and their receptor genes have been lost in the Ciona genome during the evolution of chordates ${ }^{14}$, suggesting that neuropeptides are major factors for the biological regulation in Ciona. Interestingly, our recent anatomical analyses of transgenic Ciona revealed diverse innervation of peptidergic neurons of the central nervous system to peripheral organs including ovaries, strongly suggesting that the peptidergic system directly regulates ovarian follicle growth, maturation, and ovulation in Ciona ${ }^{15,16}$. Although a few Ciona neuropeptides such as CiTK, neurotensin-like peptide-6, and CiVP have been shown to participate in ovarian functions ${ }^{11,17-19}$, the biological roles of Ciona neuropeptides and the molecular mechanisms underlying the reproductive process largely remain to be investigated.

Cholecystokinin (CCK) and gastrin are a vertebrate brain/gut peptide and a gastric hormone, respectively ${ }^{20}$. These peptides possess a signature amino acid, a sulfated tyrosine, at positions 7 and 6 from the C-terminus, and harbor a common C-terminal amidated tetrapeptide motif (Trp-Met-Asp-Phe- 
$\mathrm{NH}_{2}$ ) that is essential for receptor activation ${ }^{20}$. Synteny analysis has suggested that CCK and gastrin genes might have arisen from a common ancestral gene through a first-round whole-genome duplication event during early vertebrate evolution ${ }^{20}$. A Ciona homolog of CCK, cionin, has been isolated from the Ciona neural complex and shares the C-terminal consensus motif of vertebrate CCK and gastrin ${ }^{2,21}$ Furthermore, cionin possesses two sulfated tyrosines at positions 6 and 7 from the C-terminus ${ }^{2}$. In addition, combined with the crucial phylogenetic position of Ciona as the sister group of vertebrates, the lack of a whole-genome duplication event during the evolution of $\mathrm{Ciona}^{22}$ suggests that cionin conserves common ancestral features of CCK and gastrin.

To date, two cognate cionin receptors, CioR1 and CioR2, have been identified ${ }^{23,24}$. These receptors show high sequence homology to vertebrate CCK/gastrin receptors (CCK1R and CCK2R). CioR1 and CioR2 trigger intracellular calcium mobilization in response to cionin ${ }^{23,24}$, suggesting that these receptors are $\mathrm{G}$ protein-coupled receptors (GPCRs) coupled with $\mathrm{G}_{\mathrm{q}}$ protein. Molecular phylogenetic analysis demonstrated that both Cior1 and Cior2 genes are orthologous to the vertebrate CCKRs and were generated in the Ciona-specific evolutionary lineage ${ }^{24}$. Cior1 and Cior2 mRNAs are expressed in the neural complex, digestive organs, oral siphon, atrial siphon, and ovary ${ }^{24}$. Notably, Cior2 mRNA is highly expressed specifically in the Ciona ovary, suggesting that cionin plays pivotal roles in reproductive control $^{24}$.

In this study, we unraveled the biological functions of cionin. The present data indicated that cionin stimulates ovulation via activation of receptor tyrosine kinase (RTK) signaling pathways leading to upregulation of the expression of a matrix metalloproteinase (MMP) gene. The present study reveals a novel biological role for CCK/gastrin family peptides in the ovary, and provides clues to the evolution of the regulatory system of ovulation in chordates.

\section{Materials And Methods}

\section{Animals}

Adult Ciona intestinalis type A (synonym for Ciona robusta) ${ }^{1}$ were cultivated at the Maizuru Fisheries Research Station of Kyoto University or Misaki Marine Biological Station of the University of Tokyo and maintained in sterile artificial sea water at $18^{\circ} \mathrm{C}$.

\section{In situ hybridization}

The 3'-terminal region of Cior2 (nt 1357-1662; GenBank accession numbers AB669183 and AB863059) was inserted into the pGEM-T easy vector (Promega, Madison, WI, USA). The plasmid was linearized and used with the digoxigenin-labeled RNA labeling kit (Roche Applied Science, Penzberg, Germany). The Ciona ovaries were dissected and fixed in $4 \%$ paraformaldehyde in $0.1 \mathrm{M}$ phosphate buffer at $4{ }^{\circ} \mathrm{C}$ overnight. The fixed tissues were soaked in a refrigerated sucrose solution (30\% sucrose in PBS) until they sank. They were embedded in Super Cryoembedding Medium-L1 (Leica Microsystems Japan, Tokyo, 
Japan) and sectioned at 10- $\mu$ m thickness with a CryoStar NX70 cryostat (Thermo Fisher Scientific Inc., Waltham, MA, USA) at $-20^{\circ} \mathrm{C}$. The sections were placed onto MAS-coated slides (Matsunami Glass Ind., Ltd., Osaka, Japan). Hybridization, washing, and detection were carried out as previously reported ${ }^{4,25,26}$. No positive signals were observed when sense probes were used, confirming the specificity of hybridization. The primer set used for the Cior2 probe is listed in Table 1.

\section{Fractionation of Ciona follicles}

Fractionation of Ciona follicles was performed as previously described ${ }^{19,27}$. In brief, approximately 8 Ciona ovaries were collected and washed with artificial sea water three times. Follicles were isolated from the ovaries and were collected into a normal tissue culture dish (Corning Inc., New York, USA). The isolated Ciona follicles were fractionated using a series of stainless-steel sieves of varying particle sizes $(180,150,90,63,38$, and $20 \mu \mathrm{m}$; TOKYO SCREEN CO. LTD., Tokyo, Japan). Follicles were collected from the 150- $\mu \mathrm{m}$ sieve and transferred to a cell culture dish with a cell-repellent surface (\#628979; Greiner BioOne $\mathrm{GmbH}$, Frickenhausen, Germany). Immature/pre-ovulatory follicles (early stage II to late stage II follicles) were further isolated based on their size (average size 170-180 $\mu \mathrm{m}$ in diameter) under a stereomicroscope (Discovery V8, Carl Zeiss, Tokyo, Japan). The structure of the Ciona follicle is illustrated in Fig. S1.

\section{Evaluation of oocyte maturation and ovulation of cionin-treated follicles}

Oocyte maturation and ovulation of Ciona follicles were evaluated in vitro as previously described ${ }^{19,27}$. In brief, 16-25 follicles of stage II were randomly allocated and incubated with or without ligands including cionin, and the respective rates of germinal vesicle break-down (GVBD) in the oocytes and rupture of the outer follicular cell layer were evaluated as an indicator of oocyte maturation and ovulation after a 24-h incubation. Synthetic cionin was prepared as previously described ${ }^{24}$ and used at a final concentration of $5 \mu \mathrm{M}$ given that lower concentrations of other peptides are reportedly ineffective in Ciona ${ }^{28}$. Follicles incubated with artificial sea water were used as a control group. Follicles incubated with nonsulfated cionin (final concentration, $5 \mu \mathrm{M}$ ), which does not activate cionin receptors ${ }^{24}$, were used as a negative control group. The receptor tyrosine kinase (RTK) inhibitor, sunitinib malate (Cayman Chemical Company, MI), and MMP 2/9 inhibitor II (Calbiochem, San Diego, CA) were used for the inhibitor experiments. Sunitinib malate stock solution (1 mM) was prepared in 100\% dimethyl sulfoxide (DMSO) and diluted with artificial sea water at a final concentration of $1 \mu \mathrm{M}$. MMP2/9 inhibitor II solution (10 mM) was prepared in $100 \%$ DMSO and diluted with artificial sea water at a final concentration of $10 \mu \mathrm{M}$. Artificial sea water containing an equivalent concentration of DMSO $(0.1 \%)$ was used as a control group in the inhibitor experiments.

\section{RNA-seq of cionin-treated follicles}

Two independent sets of follicles were used for RNA-seq of the control group and cionin-treated group. Total RNA was extracted from 25-30 follicles of stage II using Sepasol-RNA I (Nacalai Tesque, Kyoto, 
Japan). The total RNA was further purified and treated with TURBO DNase as previously described ${ }^{19,27}$. A total of $200 \mathrm{ng}$ of quality-verified RNA from each sample was subjected to RNA-seq analysis using a HiSeq1500 instrument (Illumina, San Diego, CA) in rapid mode as reported previously ${ }^{29}$. The resultant reads were aligned to the Ciona genes (KH, ver. 2013), which were downloaded from the Ghost Database (http://ghost.zool.kyoto-u.ac.jp/cgi-bin/gb2/gbrowse/kh/). The new Ghost Database

(http://ghost.zool.kyoto-u.ac.jp/default_ht.html) was also referred to for new accession numbers ${ }^{30}$. The expression level of each gene was calculated as the gene-specific reads per kilobase per million total reads (RPKM). Genes satisfying the condition of read number $>10$ or RPKM value $>0.5$ were selected to calculate the RPKM ratio. Genes satisfying the condition of RPKM ${ }^{\text {cionin }}$ value/RPKM ${ }^{\text {control }}$ value $>1.5$ were defined as those upregulated in the cionin-treated follicles. Genes satisfying the condition of $\mathrm{RPKM}^{\text {cionin }}$ value/RPKM ${ }^{\text {control }}$ value $<0.67$ were defined as those downregulated in the cionin-treated follicles. Genes satisfying the condition of $1.12>\mathrm{RPKM}^{\text {cionin }}$ value/RPKM ${ }^{\text {control }}$ value $>0.90$ were defined as those unchanged in the cionin-treated follicles. Among these, 985 genes were selected for upregulated genes and downregulated genes, and 1970 genes were selected for unchanged genes for gene ontology (GO) analysis. Subsequently, putative genes for transcripts were annotated based on a homology search of the NCBI database under the condition of evalue $<10^{-3}$ using Blast2GO software (version 4.1.9) with default parameters ${ }^{31}$. GO analyses were performed using Blast2GO software and visualized with Cytoscape software as previously reported ${ }^{29}$.

\section{Real-time PCR analysis of cionin-treated follicles}

Total RNA was extracted from 25-30 follicles of stage II as described above. A 300-ng aliquot of DNasetreated total RNA was used for first-strand CDNA synthesis. Real-time PCR was performed using a CFX96 Touch Real-Time PCR Detection System (Bio-Rad Laboratories, Inc., Hercules, CA) as previously described ${ }^{19,29}$. The primers used for real-time PCR analyses are listed in Table 1. Gene expression levels were normalized to the expression of the ubiquitin-associated domain containing one gene (CiUbac1, KH.L133.5 or KY.Chr12.25), which was found to be constitutively expressed in the follicles as previously described $^{19}$.

\section{Statistical analysis}

Results are shown as means \pm SEM. Data were analyzed by Student's $t$ test or one-way ANOVA followed by Tukey's post hoc test. Differences were accepted as significant for $P<0.05$ or $P<0.01$.

\section{Results}

\section{Localization of Cior2 mRNA in the Ciona ovary}

To investigate the biological functions of cionin, we first analyzed the localization of cionin receptors in the ovary. Cior 2 mRNA was present exclusively in inner follicular cells, but not in oocytes or test cells, of pre-ovulatory follicles including stage II (Figs. 1, S1). These results indicate that the inner follicular cells 
of the pre-ovulatory follicles are the major target of cionin in the ovary. In addition, no positive signal was detected using sense probes (Fig. 1).

\section{Effects of cionin on the ovulation and maturation of follicles}

Pre-ovulatory follicles (average sizes of 170-180 $\mu \mathrm{m}$ in diameter), which were CioR2-positive (Fig. 1), were incubated with cionin for $24 \mathrm{~h}$, and the ratios of ovulation and GVBD rates were estimated. The ovulation rate of cionin-treated follicles was significantly increased $(9.2 \pm 2.43 \%$ to $37.86 \pm 5.51 \%)$, compared with the control follicles (Fig. 2, Supplemental Video). In contrast, the GVBD rate of cionintreated follicles was not significantly altered (Fig. 2 and Supplemental Video). Nonsulfated cionin, which fails to activate cionin receptors ${ }^{24}$, significantly increased neither ovulation nor GVBD rates after the 24-h incubation, proving that cionin specifically stimulated the ovulation (Fig. 2 and Supplemental Video).

\section{Transcriptomic And Real-time Pcr Analyses Of Cionin-treated Follicles}

To investigate the mechanism underlying the cionin-induced ovulation, we performed RNA-seq and GO term analysis. RNA-seq for the control and cionin-treated groups yielded 12 million reads, respectively, for 101 single-end reads. Totals of 49,011 and 48,436 transcripts were mapped from reads of the control group and cionin-treated group, respectively. The resultant fastq files were deposited in the SRA database (SRA accession numbers: SRR10484994 and SRR10484993 for the control group, SRR10484992 and SRR10484991 for the cionin-treated group). GO term analysis of the fractionated follicles showed that cionin affected the expression of genes involved in the following: regulation of biological process, response to stimulus, locomotion, development/morphogenesis, single-organism cellular process, transport, and metabolic process (Fig. 3). Among these genes, RTK-like orphan receptor (Rora), fibrillar collagen (Fco/1), transmembrane $\gamma$-carboxyglutamic acid protein 3 (G/a3), and MMP (CiMmp2/9/13) were considerably increased in the cionin-treated groups, compared with the control groups (Table S1). Quantitative PCR further confirmed 1.6-fold, 2.8-fold, 7.5-fold, and 5.5-fold upregulation of the expressions of Rora, FCol1, Gla3, and CiMmp2/9/13 in the cionin-treated groups (Fig. 4).

\section{Key roles for RTK and MMP signaling in ovulation by cionin}

As shown in Fig. 4, cionin upregulated the expression of RTK signaling-related genes, Rora, Fcol1, and Gla3. We thus examined whether these RTK signaling-related genes are involved in the cionin-induced ovulation. Of particular interest is that the ovulation rate of the cionin-treated follicles was markedly decreased by an inhibitor of RTK-related family proteins including Rora, sunitinib malate, after the 24-h incubation $(67.63 \pm 2.59 \%$ to $30.3 \pm 4.01 \%$ ), whereas sunitinib malate did not affect the rate of GVBD (Fig. 5). These results indicated that RTK signaling participates in ovulation induced by cionin. Subsequently, we also examined the effect of an MMP inhibitor on the ovulation rate of cionin-treated follicles, given that cionin also significantly increased the expression of the CiMmp2/9/13 gene (Fig. 4). As shown in Fig. 6, the ovulation of the cionin-treated follicles was significantly suppressed by MMP 2/9 inhibitor II after the 24-h incubation (39.29 $\pm 7.14 \%$ to $11.57 \pm 4.31 \%$ ). In contrast, MMP 2/9 inhibitor II did not alter the rate of GVBD (Fig. 6). In combination, we concluded that MMP2/9/13 was responsible for 
cionin-induced ovulation. Furthermore, real-time PCR demonstrated that the administration of sunitinib malate arrested the upregulation of the CiMmp2/9/13 gene by cionin, confirming that RTKs play an essential role in the upregulation of the expression of CiMmp2/9/13 by cionin (Fig. 7). Altogether, these results verified that cionin induced ovulation via upregulation of the RTK-signaling-MMP activation pathway (Fig. 8).

\section{Discussion}

CCK is a major vertebrate brain/gut peptide and plays multiple roles in the regulation of food intake, appetite, learning, and emotion ${ }^{20}$. In contrast, the endogenous role of the Ciona CCK homolog, cionin, has not been investigated. In the present study, we identified cionin as a direct inducer of ovulation and verified the underlying fundamental molecular mechanisms. The cionin gene is expressed almost exclusively in the cerebral neurons ${ }^{2,12,24}$, and its cognate receptor, CioR2 ${ }^{24}$, was shown to be localized to the inner follicular cells of stage II follicles in the ovary (Fig. 1). Moreover, the Ciona ovary is innervated by multiple neuropeptidergic neurons in the cerebral ganglion ${ }^{15}$. Combined with these findings, the present study indicates that cionin is produced in and secreted from the cerebral ganglion to the ovary, and directly acts on inner follicular cells followed by the induction of ovulation (Fig. 2). To the best of our knowledge, this is the first evidence of a biological role of CCK family peptides in the regulation of ovulation in all animals.

Follicular growth, maturation, and ovulation are crucial processes in the development of an animal, and thus these reproductive processes are expected to be regulated by multiple signaling pathways involving various endogenous factors. In the present study, cionin was shown to activate ovulation via activation of CiMMP2/9/13 (Figs. 2, 6, Supplemental Video). Recently, we provided evidence that the Ciona VP homolog, CiVP, induces ovulation via the activation of CiMMP2/9/13 ${ }^{19}$. In addition, MMPs are highly conserved collagenases that are known to play pivotal roles in ovulation in teleosts ${ }^{19,32}$, suggesting that the induction of gene expression and enzymatic activity of MMPs are evolutionarily conserved in the ovulation process of chordates. In contrast, distinct differences in activation between cionin- and CiVPsignaling pathways were verified. First, Cior2 was expressed in inner follicular cells (Fig. 1), whereas the CiVP receptor gene (CiVpr) is expressed in oocytes ${ }^{19}$. Second, cionin was shown to stimulate the RTK signaling pathway for the induction of CiMMP2/9/13 (Figs. 4, 5), whereas CiVP upregulates CiMmp2/9/13 via mitogen-activated protein kinase (MAPK) signaling pathways ${ }^{19}$. Third, cionin is responsible for only ovulation (Fig. 2, Supplemental Video), whereas CiVP participates in both oocyte maturation and ovulation ${ }^{19}$. Altogether, the present study revealed that activation of CiMMP2/9/13 for ovulation is regulated by multiple distinct endogenous factors and signaling pathways. The presence of such multiple regulatory systems also sheds light on the biological significance of CiMMP2/9/13 in ovulation in Ciona. In addition, the inner follicular cells of Ciona are known to secrete self-sterility factors and establish the self-discrimination of oocytes during maturation ${ }^{33,34}$. Consequently, the present study also verified that inner follicular cells are responsible for not only self-discrimination but also the ovulation of follicles. 
A striking feature of the regulatory mechanism underlying Ciona ovulation is that RTK signaling factors participate in cionin-induced ovulation (Figs. 3,4). RORa is a member of the RTK family in vertebrates ${ }^{35}$. GLA3 is a vertebrate transmembrane gamma-carboxyglutamic acid protein that has multiple epidermal growth factor (EGF) domains ${ }^{36}$ and is presumed to be a ligand of another RTK, $\mathrm{Axl}^{36}$. Fibrillar collagens including FCOL1 activate discoidin domain receptors (DDRs), which also belongs to the RTK family 37,38 . Furthermore, an inhibitor of multiple RTKs, sunitinib malate, was shown to inhibit DDRs ${ }^{39}, \mathrm{ROR}^{40}$, and $\mathrm{Axl}^{40}$, and significantly inhibited the increase in the ovulation rate of cionin-treated follicles (Fig. 5). In vertebrates, various GPCRs, including CCK receptors, activate RTKs via various signaling pathways including intracellular calcium, protein kinase $C$, and Src protein kinase via coupling with $G_{q}$ protein ${ }^{41-43}$. These findings are compatible with the present study demonstrating that CioR2, coupled with $\mathrm{G}_{\mathrm{q}}{ }^{24}$, stimulates RTK signaling via intracellular calcium in response to cionin in the follicles. Consequently, the present study provides evidence that cionin stimulates ovulation via upregulation of the CioR2-RTK signaling pathway in follicles (Fig. 8). Investigation of the functional relationship among RORa, GLA3 and FCOL1 awaits further study.

It is also noteworthy that sunitinib malate inhibits ovulation but does not affect follicle growth or maturation in mice ${ }^{44}$. In addition, RTK signaling lies downstream of the luteinizing hormone surge and is essential to initiate the ovulatory response in mammals ${ }^{45}$. These results suggest that RTKs are involved in ovulation in mammals, although no factors inducing RTK signaling in mammalian ovulation have been identified. In other words, CCK may also play some roles in ovulatory processes in mammals.

We also revealed upregulation of CiMMP2/9/13 via activation of RTK signaling, given that the cionininduced CiMMP2/9/13 gene expression was markedly suppressed by sunitinib malate (Fig. 6). This signaling in Ciona is compatible with previous findings indicating that MMPs are induced by RTKs, including discoidin domain receptors and vascular endothelial growth factor in mammalian smooth muscle cells or human placenta choriocarcinoma cell lines ${ }^{46,47}$, suggesting that the RTK-MMP regulatory pathway also functions in the ovaries in mammals, although neither the proteases essential for mammalian ovulation nor the signaling molecules that upregulate the RTK pathways have been investigated. Moreover, the $C c k 2 r$ gene is expressed in the ovaries of adult mice ${ }^{48}$ (BioProject accession number: PRJNA66167), and CCK2R has been reported to induce the expression of an RTK-related gene in rat gastric epithelial cells ${ }^{49}$. Altogether, these findings suggest that CCK/gastrin-RTK signaling is also responsible for the regulation of ovulation in vertebrates, including mammals, and that the ovulation mechanism regulated by the CCK family peptides-RTK signaling-MMP pathway, elucidated in the present study, is conserved during the evolution of chordates. The investigation of the biological roles of $\mathrm{CCK} /$ gastrin in ovulation or other ovarian functions in vertebrates is underway.

In conclusion, we have substantiated that the Ciona CCK homolog, cionin, induces ovulation by upregulating MMP via the RTK signaling pathway in Ciona, the closest relative of vertebrates. The present study has clarified not only the novel regulatory mechanisms underlying ovulation in Ciona and a novel 
biological role for CCK/gastrin family peptides in chordates, but also paved the way for understanding the biological roles and evolution of neuropeptidergic regulation of ovarian functions in chordates.

\section{Declarations}

\section{Acknowledgements}

We gratefully acknowledge Prof. Shigetada Nakanishi for his fruitful comments on the manuscript. We thank the National Bio-Resource Project for providing ascidians. This study was supported by Grants-inAid for Scientific Research from the Ministry of Education, Culture, Sports, Science and Technology (MEXT), Japan (JP16K07430 to HS, 26840108 to TO, JP16K18581 and JP17J10624 to SM).

\section{Author Contributions}

T.O. and H.S. designed the study; T.O., N.M., A.S., S.M., and H.S. performed the research; T.O., A.S., S.M., and H.S. wrote, reviewed, and edited the paper; H.S. supervised the study.

\section{Data availability}

The transcriptome data are publicly available under accession numbers SRR10484994 and SRR10484993 for the control group, and SRR10484992 and SRR10484991 for the cionin-treated group in the NCBI SRA database.

\section{Additional Information}

Competing Interests: The authors declare no competing interests.

\section{References}

1. Brunetti, R. et al. Morphological evidence that the molecularly determined Ciona intestinalis type A and type B are different species: Ciona robusta and Ciona intestinalis. J Zoolog Syst Evol Res. 53, 186-193 (2015).

2. Johnsen, A. H. \& Rehfeld, J. F. Cionin: a disulfotyrosyl hybrid of cholecystokinin and gastrin from the neural ganglion of the protochordate Ciona intestinalis. J Biol Chem. 265, 3054-3058 (1990).

3. Adams, B. A. et al. Six novel gonadotropin-releasing hormones are encoded as triplets on each of two genes in the protochordate, Ciona intestinalis. Endocrinology. 144, 1907-1919 (2003).

4. Satake, H. et al. Tachykinin and tachykinin receptor of an ascidian, Ciona intestinalis: evolutionary origin of the vertebrate tachykinin family. J Biol Chem. 279, 53798-53805 (2004).

5. Olinski, R. P., Dahlberg, C., Thorndyke, M. \& Hallböök, F. Three insulin-relaxin-like genes in Ciona intestinalis. Peptides. 27, 2535-2546 (2006).

6. Olinski, R. P., Lundin, L. G. \& Hallböök, F. Conserved synteny between the Ciona genome and human paralogons identifies large duplication events in the molecular evolution of the insulin-relaxin gene 
family. Mol Biol Evol. 23, 10-22 (2006).

7. Kawada, T., Sekiguchi, T., Itoh, Y., Ogasawara, M. \& Satake, H. Characterization of a novel vasopressin/oxytocin superfamily peptide and its receptor from an ascidian, Ciona intestinalis. Peptides. 29, 1672-1678 (2008).

8. Kawada, T. et al. A novel inhibitory gonadotropin-releasing hormone-related neuropeptide in the ascidian, Ciona intestinalis. Peptides. 30, 2200-2205 (2009).

9. Sekiguchi, T. et al. Calcitonin in a protochordate, Ciona intestinalis - the prototype of the vertebrate calcitonin/calcitonin gene-related peptide superfamily. FEBS J. 276, 4437-4447 (2009).

10. Kawada, T., Sekiguchi, T., Sakai, T., Aoyama, M. \& Satake, H. Neuropeptides, hormone peptides, and their receptors in Ciona intestinalis: an update. Zool Sci. 27, 134-153 (2010).

11. Kawada, T. et al. Peptidomic analysis of the central nervous system of the protochordate, Ciona intestinalis: homologs and prototypes of vertebrate peptides and novel peptides. Endocrinology. 152, 2416-2427 (2011).

12. Satake, H., Sekiguchi, T., Sakai, T., Aoyama, M. \& Kawada, T. Endocrinology and neuroendocrinology of protochordates: Evolutionary views and potentials as new model organisms. Recent Res Devel Endocrinol. 5, 1-19 (2013).

13. Shiraishi, A. et al. Repertoires of $G$ protein-coupled receptors for Ciona-specific neuropeptides. Proc Natl Acad Sci USA. 116, 7847-7856 (2019).

14. Dehal, P. et al. The draft genome of Ciona intestinalis: insights into chordate and vertebrate origins. Science. 298, 2157-2167 (2002).

15. Osugi, T., Sasakura, Y. \& Satake, H. The nervous system of the adult ascidian Ciona intestinalis Type A (Ciona robusta): Insights from transgenic animal models. PLoS One. 12, e0180227 (2017).

16. Osugi, T., Sasakura, Y. \& Satake, H. The ventral peptidergic system of the adult ascidian Ciona robusta (Ciona intestinalis Type A) insights from a transgenic animal model. Sci Rep. 10, 1892 (2020).

17. Aoyama, M. et al. A novel biological role of tachykinins as an up-regulator of oocyte growth: identification of an evolutionary origin of tachykininergic functions in the ovary of the ascidian, Ciona intestinalis. Endocrinology. 149, 4346-4356 (2008).

18. Aoyama, M., Kawada, T. \& Satake, H. Localization and enzymatic activity profiles of the proteases responsible for tachykinin-directed oocyte growth in the protochordate, Ciona intestinalis. Peptides. 34, 186-192 (2012).

19. Matsubara, S., Shiraishi, A., Osugi, T., Kawada, T. \& Satake, H. The regulation of oocyte maturation and ovulation in the closest sister group of vertebrates. eLife 8, pii:e49062(2019).

20. Dupré, D. \& Tostivint, H. Evolution of the gastrin-cholecystokinin gene family revealed by synteny analysis. Gen Comp Endocrinol. 195, 164-173 (2014).

21. Miller, L. J. \& Gao, F. Structural basis of cholecystokinin receptor binding and regulation. Pharmacol Ther. 119, 83-95 (2008). 
22. Dehal, P. \& Boore, J. L. Two rounds of whole genome duplication in the ancestral vertebrate. PLoS Biol. 3, e314 (2005).

23. Nilsson, I. B., Svensson, S. P. \& Monstein, H. J. Molecular cloning of a putative Ciona intestinalis cionin receptor, a new member of the CCK/gastrin receptor family. Gene. 323, 79-88 (2003).

24. Sekiguchi, T., Ogasawara, M. \& Satake, H. Molecular and functional characterization of cionin receptors in the ascidian, Ciona intestinalis: the evolutionary origin of the vertebrate cholecystokinin/gastrin family. J Endocrinol. 213, 99-106 (2012).

25. Satake, H., Takuwa, K., Minakata, H. \& Matsushima, O. Evidence for conservation of the vasopressin/oxytocin superfamily in Annelida. J Biol Chem. 274, 5605-5611 (1999).

26. Osugi, T. et al. Evolutionary origin of the structure and function of gonadotropin-inhibitory hormone: insights from lampreys. Endocrinology. 153, 2362-2374 (2012).

27. Matsubara, S., Shiraishi, A., Osugi, T., Kawada, T. \& Satake, H. Fractionation of ovarian follicles and in vitro oocyte maturation and ovulation assay of Ciona intestinalis Type A. Bio protocol. 10, e3577 (2020).

28. Kamiya, C. et al. Nonreproductive role of gonadotropin-releasing hormone in the control of ascidian metamorphosis. Dev Dyn. 243, 1524-1535 (2014).

29. Kawada, T., Shiraishi, A., Aoyama, M. \& Satake, H. Transcriptomes of the premature and mature ovaries of an ascidian, Ciona intestinalis. Front Endocrinol (Lausanne). 8, 88 (2017).

30. Satou, Y. et al. A nearly complete genome of Ciona intestinalis Type A (C. robusta) reveals the contribution of inversion to chromosomal evolution in the genus Ciona. Genome Biol Evol. 11, 31443157 (2019).

31. Conesa, A. et al. Blast2GO: a universal tool for annotation, visualization and analysis in functional genomics research. Bioinformatics. 21, 3674-3676 (2005).

32. Ogiwara, K., Takano, N., Shinohara, M., Murakami, M. \& Takahashi, T. Gelatinase A and membranetype matrix metalloproteinases 1 and 2 are responsible for follicle rupture during ovulation in the medaka. Proc Natl Acad Sci USA. 102, 8442-8447 (2005).

33. Pinto, M. R., De Santis, R., Marino, R. \& Usui, N. Specific induction of self-discrimination by follicle cells in Ciona intestinalis oocytes. Develop Growth Differ. 37, 287-291 (1995).

34. Marino, R., De Santis, R., Giuliano, P. \& Pinto, M. R. Follicle cell proteasome activity and acid extract from the egg vitelline coat prompt the onset of self-sterility in Ciona intestinalis oocytes. Proc Nat/ Acad Sci USA. 96, 9633-9636 (1999).

35. Auger, H. et al.. Similar regulatory logic in Ciona intestinalis for two Wnt pathway modulators, ROR and SFRP-1/5. Dev Biol. 329, 364-373 (2009).

36. Kulman, J. D. et al.. Vitamin K-dependent proteins in Ciona intestinalis, a basal chordate lacking a blood coagulation cascade. Proc Natl Acad Sci USA. 103, 15794-15799 (2006).

37. Vogel, W., Gish, G. D., Alves, F. \& Pawson, T. The discoidin domain receptor tyrosine kinases are activated by collagen. Mol Cell. 1, 13-23 (1997). 
38. Shrivastava, A. et al. An orphan receptor tyrosine kinase family whose members serve as nonintegrin collagen receptors. Mol Cell. 1, 25-34 (1997).

39. Kitagawa, D. et al. Activity-based kinase profiling of approved tyrosine kinase inhibitors. Genes Cells. 18, 110-122 (2013).

40. Martinho, O. et al. In vitro and in vivo analysis of RTK inhibitor efficacy and identification of its novel targets in glioblastomas. Transl Oncol 6, 187-196(2013).

41. Wetzker, R. \& BÓ§hmer, F. D. Transactivation joins multiple tracks to the ERK/MAPK cascade. Nat Rev Mol Cell Biol. 4, 651-657 (2003).

42. Shah, B. H. \& Catt, K. J. GPCR-mediated transactivation of RTKs in the CNS: mechanisms and consequences. Trends Neurosci. 27, 48-53 (2004).

43. Piiper, A. et al. Cholecystokinin stimulates extracellular signal-regulated kinase through activation of the epidermal growth factor receptor, Yes, and protein kinase C. Signal amplification at the level of Raf by activation of protein kinase Cepsilon. J Biol Chem. 278, 7065-7072 (2003).

44. Bernard, V. et al. The tyrosine kinase inhibitor sunitinib affects ovulation but not ovarian reserve in mouse: a preclinical study. PLoS One. 11, e0152872 (2016).

45. Robker, R. L., Hennebold, J. D. \& Russell, D. L. Coordination of ovulation and oocyte maturation: a good egg at the right time. Endocrinology. 159, 3209-3218 (2018).

46. Hou, G., Vogel, W. F. \& Bendeck, M. P. Tyrosine kinase activity of discoidin domain receptor 1 is necessary for smooth muscle cell migration and matrix metalloproteinase expression. Circ Res. 90, 1147-1149 (2002).

47. Zhang, J. et al. Effects of fibronectin, VEGF and angiostatin on the expression of MMPs through different signaling pathways in the JEG-3 cells. Am J Reprod Immunol. 50, 273-285 (2003).

48. Lay, J. M., Jenkins, C., Friis-Hansen, L. \& Samuelson, L. C. Structure and developmental expression of the mouse CCK-B receptor gene. Biochem Biophys Res Commun. 272, 837-842 (2000).

49. Zeng, Q., Ou, L., Wang, W. \& Guo, D. Y. Gastrin, cholecystokinin, signaling, and biological activities in cellular processes. Front Endocrinol (Lausanne). 11, 112 (2020).

\section{Tables}

Due to technical limitations, table 1 is only available as a download in the Supplemental Files section.

\section{Figures}




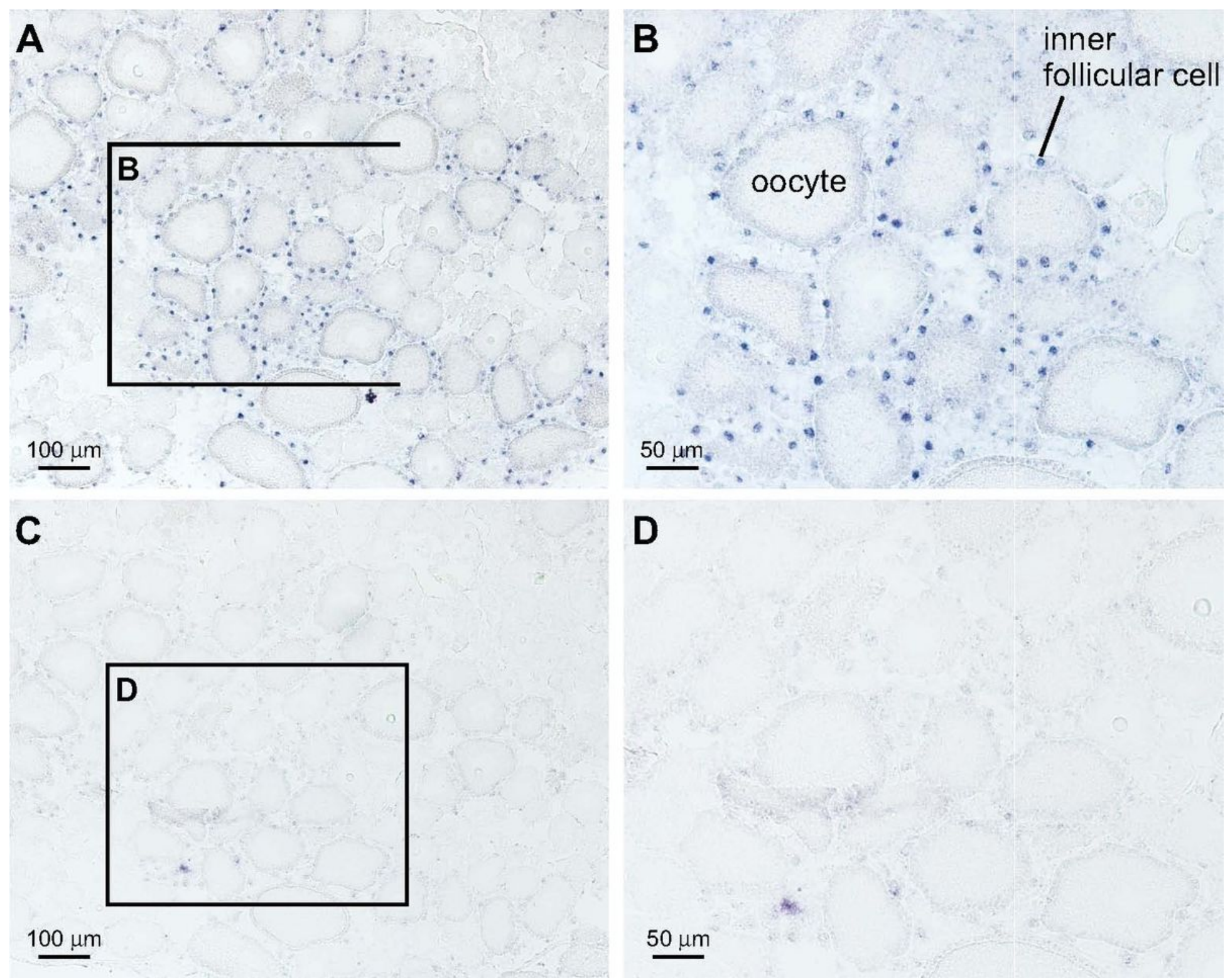

Figure 1

Localization of CioR2 mRNA in the ovary. (A) In situ hybridization of CioR2 mRNA in the ovary. Positive signals are shown in the inner follicular cells of stage II follicles. (B) Magnification of the rectangular region in (A). (C) Negative control using the sense RNA probe. No hybridization signal was observed. (D) Magnification of the rectangular region in (C). 


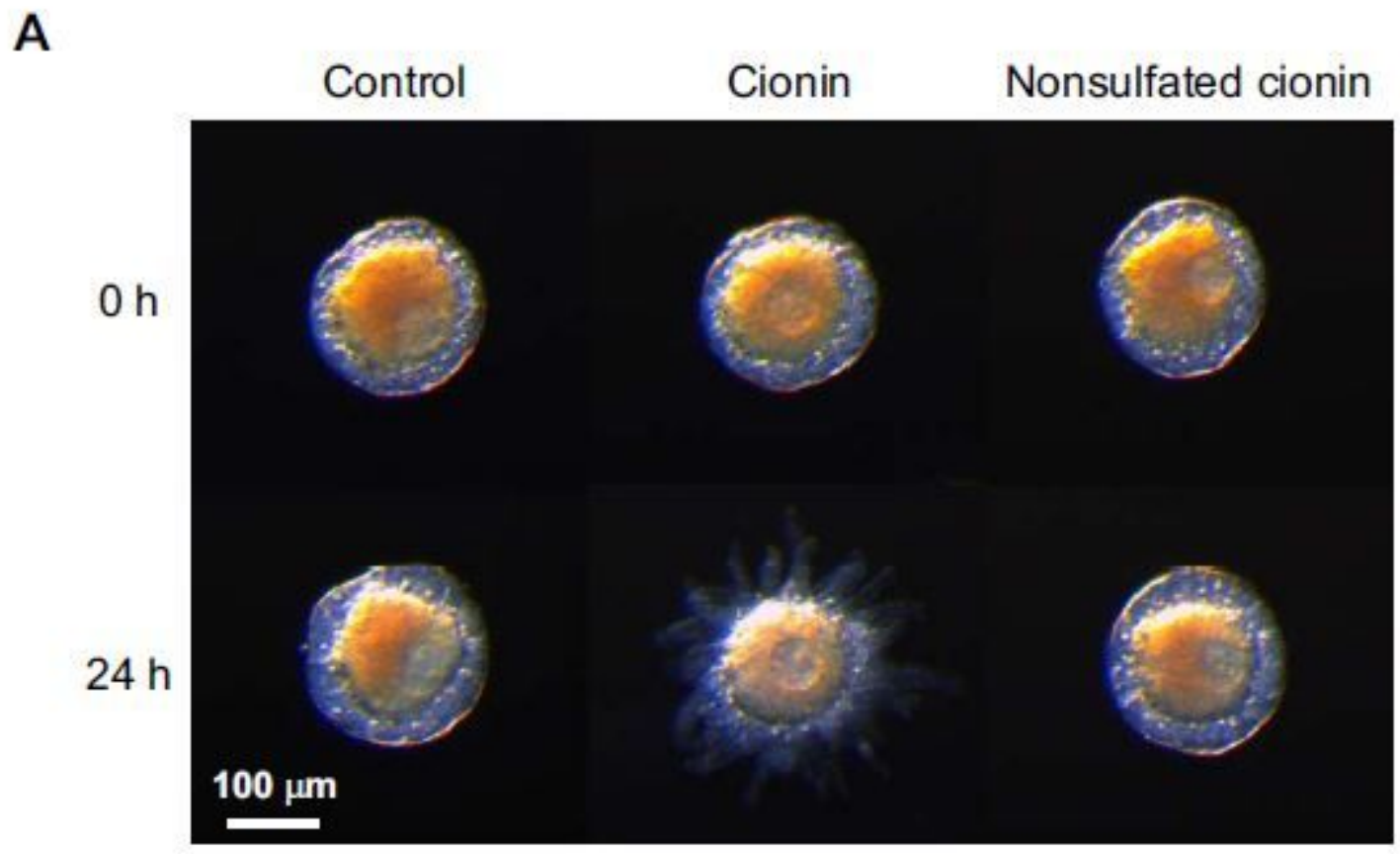

\section{B}

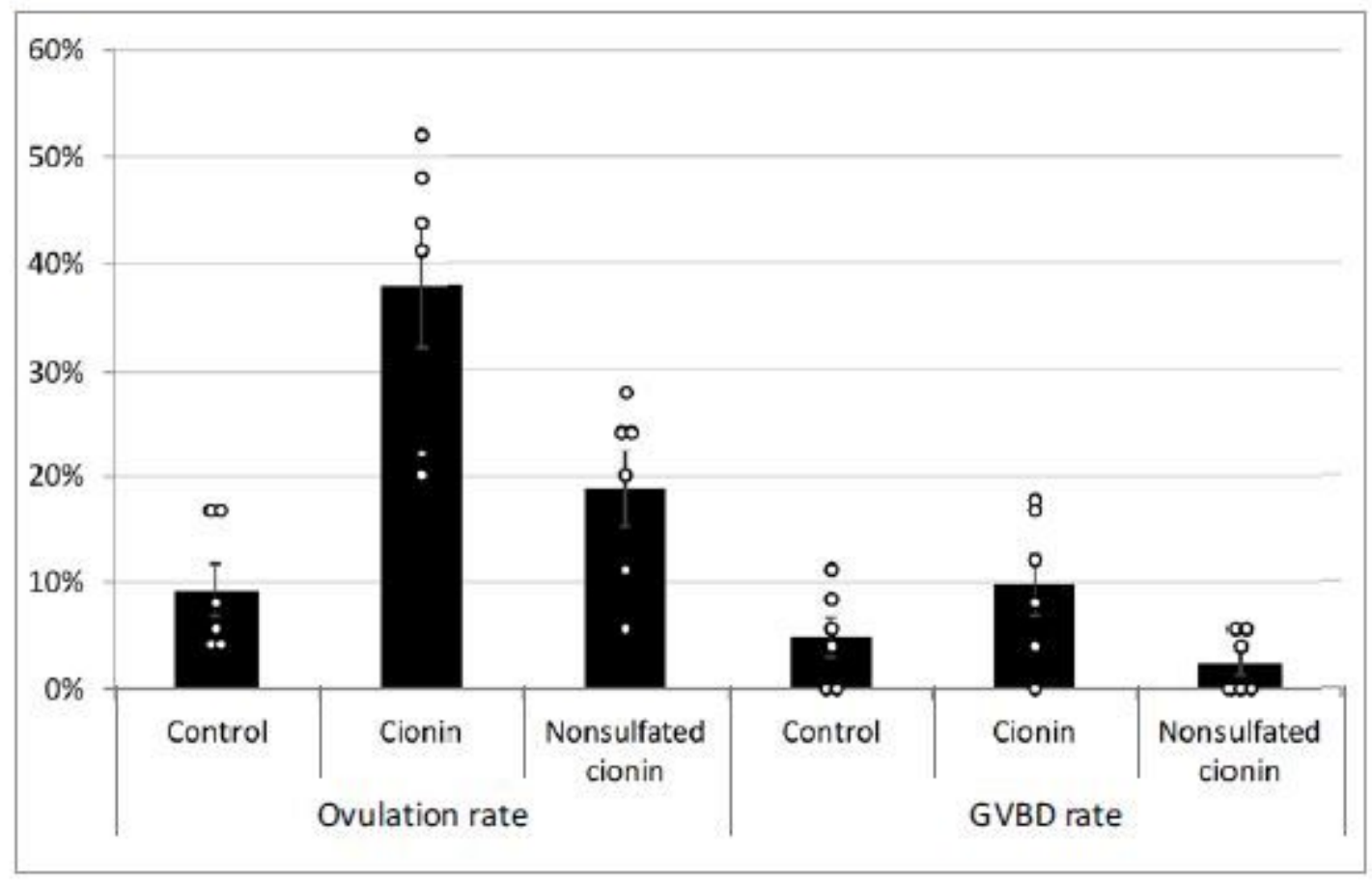

\section{Figure 2}

Effects of cionin on the ovulation and GVBD of follicles. (A) Representative images of follicles before and after treatment with $5 \mu \mathrm{M}$ cionin or nonsulfated cionin for $24 \mathrm{~h}$. Ovulation of stage II follicles was enhanced in the presence of cionin, whereas it was not in the presence of nonsulfated cionin. (B) Ovulation and GVBD rates were calculated after a 24-h incubation. Cionin significantly increased the 
ovulation rate but not the GVBD rate. Results are means \pm SEM $(n=6) .{ }^{*}, P<0.05$ cionin vs. nonsulfated cionin; and **, $\mathrm{P}<0.01$ control vs. cionin (one-way ANOVA followed by Tukey's post hoc test).

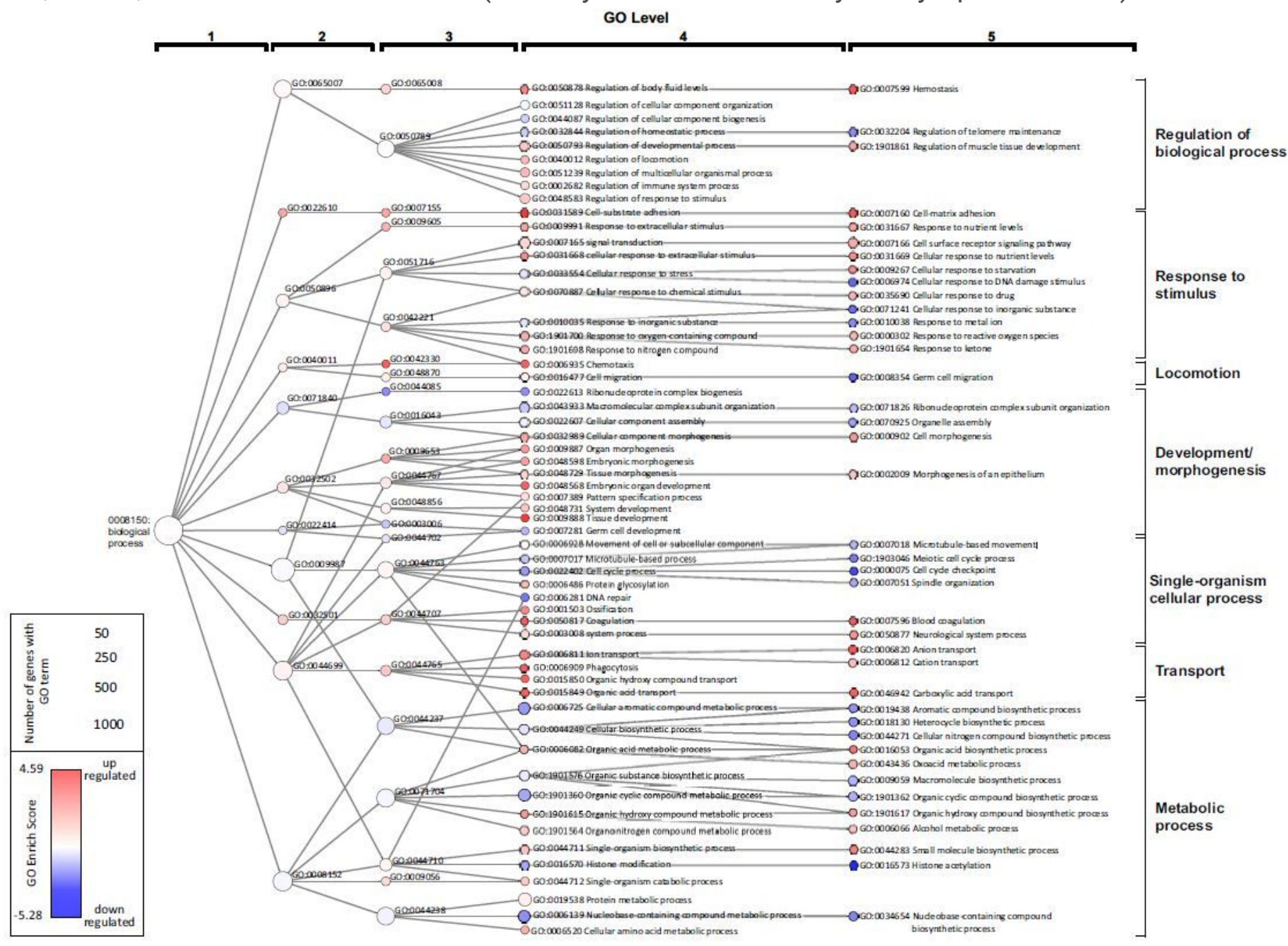

\section{Figure 3}

Gene ontology (GO) enrichment analysis of increased and decreased genes in the cionin-treated follicles. The GO category shown is "biological process" (BP). Each node represents a GO term. The circle size of each node indicates the number of genes with that GO term. The color of each node represents the enrichment score. Red and blue colored nodes indicate high enrichment scores of increased and decreased genes in cionin-treated follicles, respectively. Edges represent "is_a" connections between GO terms. 

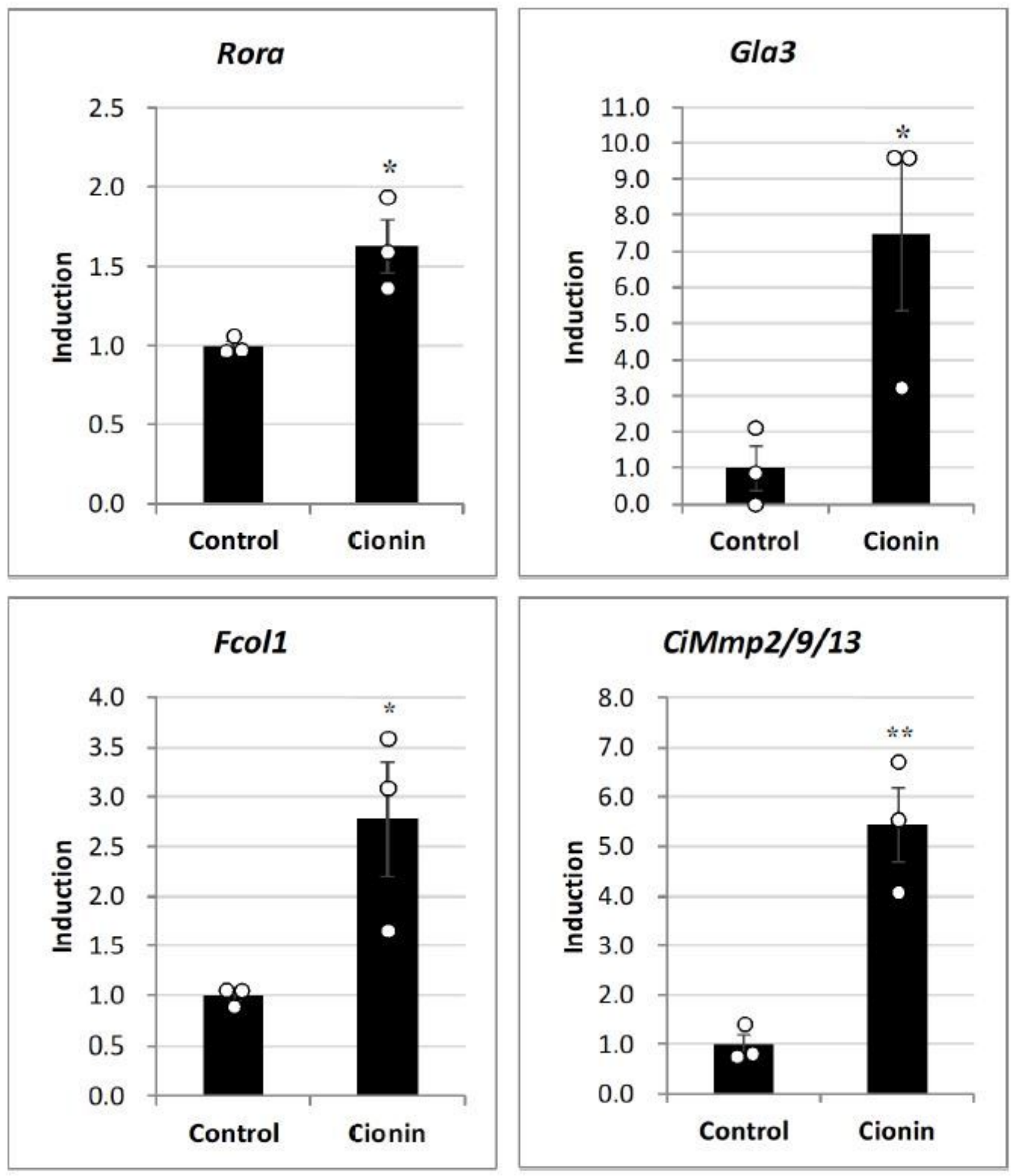

Figure 4

Real-time PCR analysis of cionin-treated follicles. Cionin stimulated the expression of Rora, Gla3, Fcol1, and CiMmp2/9/13 genes after a 24-h incubation. Data from three independent experiments $(n=3)$ were analyzed by Student's $t$ test and are shown as means \pm SEM. * $P<0.05$; $* *, P<0.01$, control vs. cionin. 
A

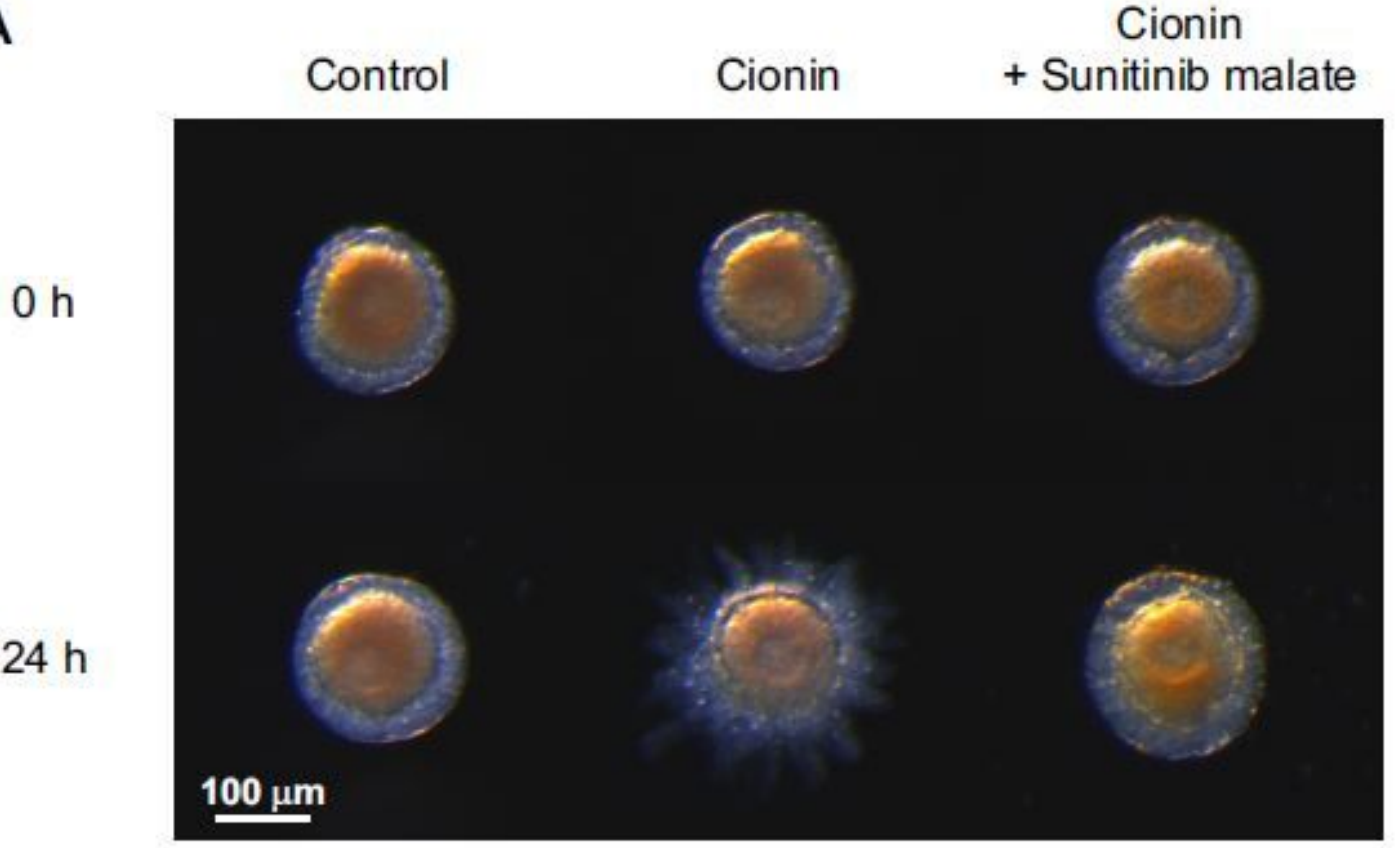

B

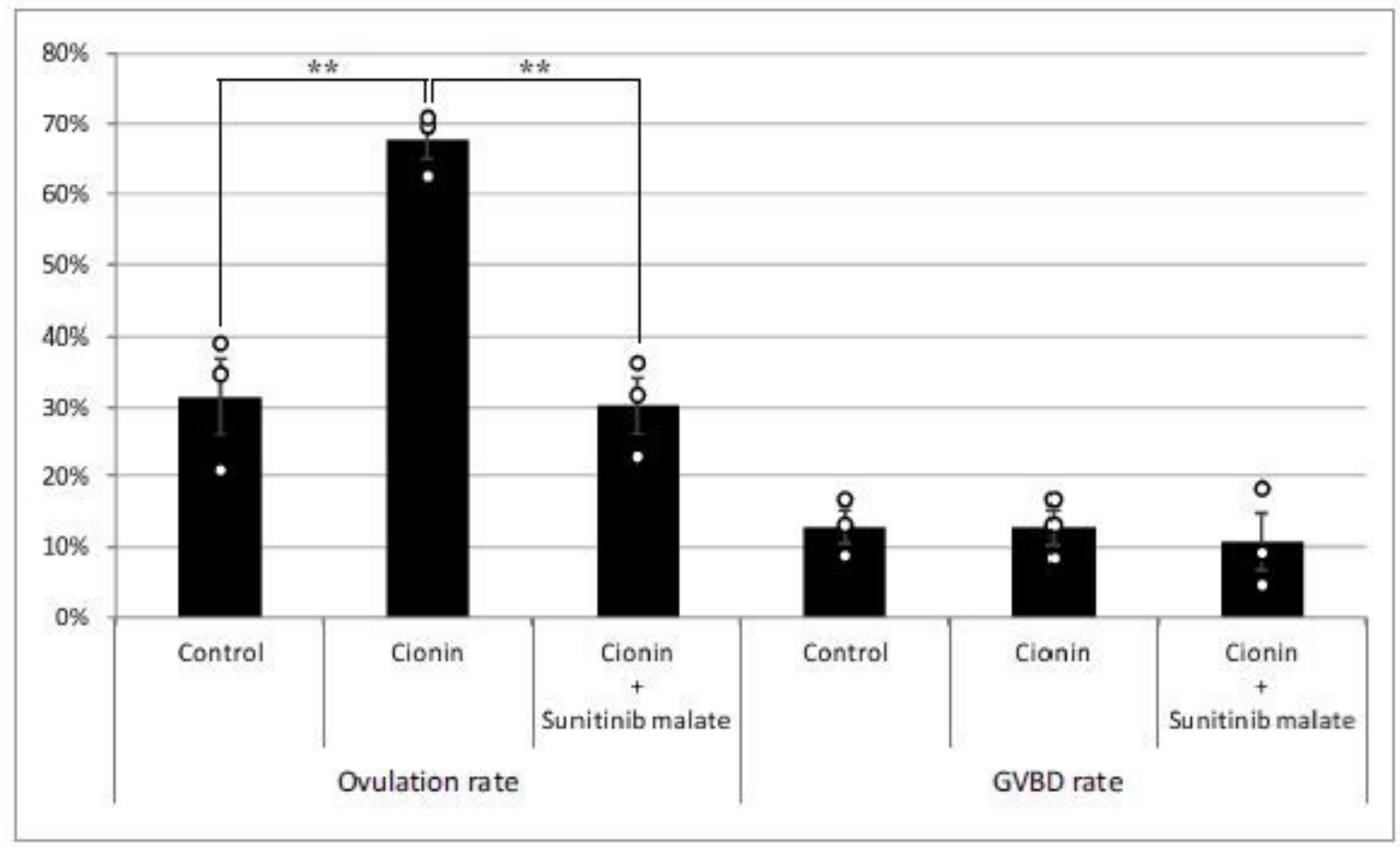

\section{Figure 5}

Effect of the receptor tyrosine kinase inhibitor, sunitinib malate, on the ovulation and GVBD of cionintreated follicles. (A) Representative images of follicles before and after treatment with $5 \mu \mathrm{M}$ cionin or 5 $\mu \mathrm{M}$ cionin and $1 \mathrm{mM}$ sunitinib malate for $24 \mathrm{~h}$. Cionin-induced ovulation of stage II follicles was disrupted by sunitinib malate. (B) Ovulation and GVBD rates were calculated after the 24-h incubation. Sunitinib malate significantly inhibited ovulation in the cionin-treated follicles. Sunitinib malate did not affect 
GVBD rate. Results are means $\pm \operatorname{SEM}(n=3)$ * ${ }^{\star *}, P<0.01$ control vs. cionin or cionin vs. cionin + sunitinib malate (one-way ANOVA followed by Tukey's post hoc test).

A

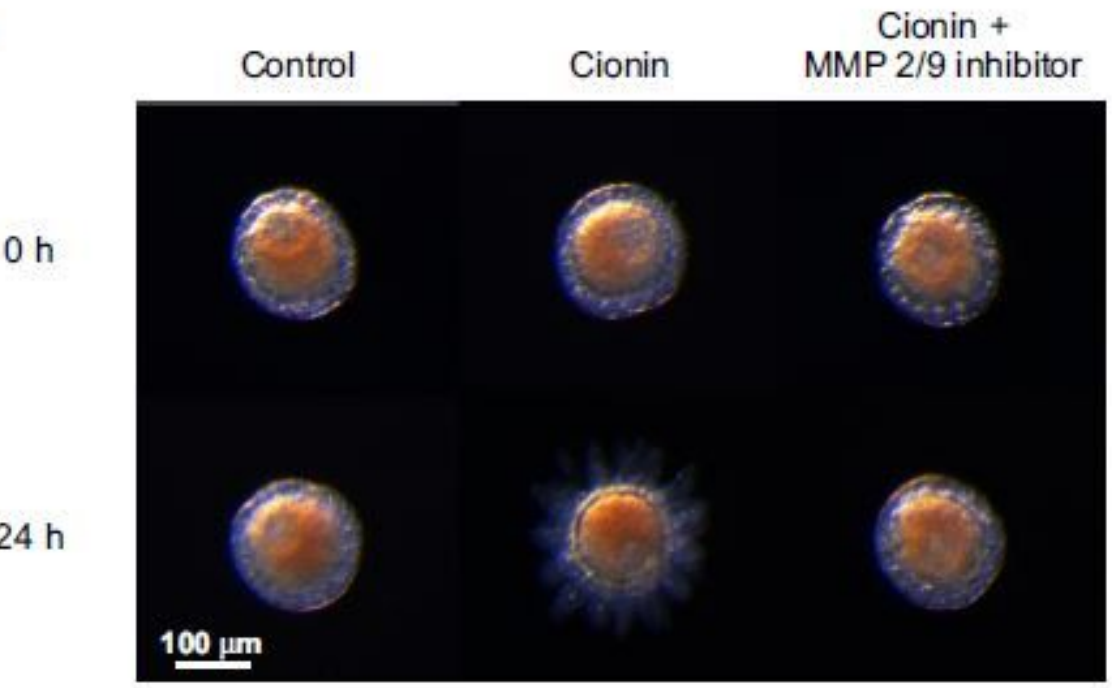

B

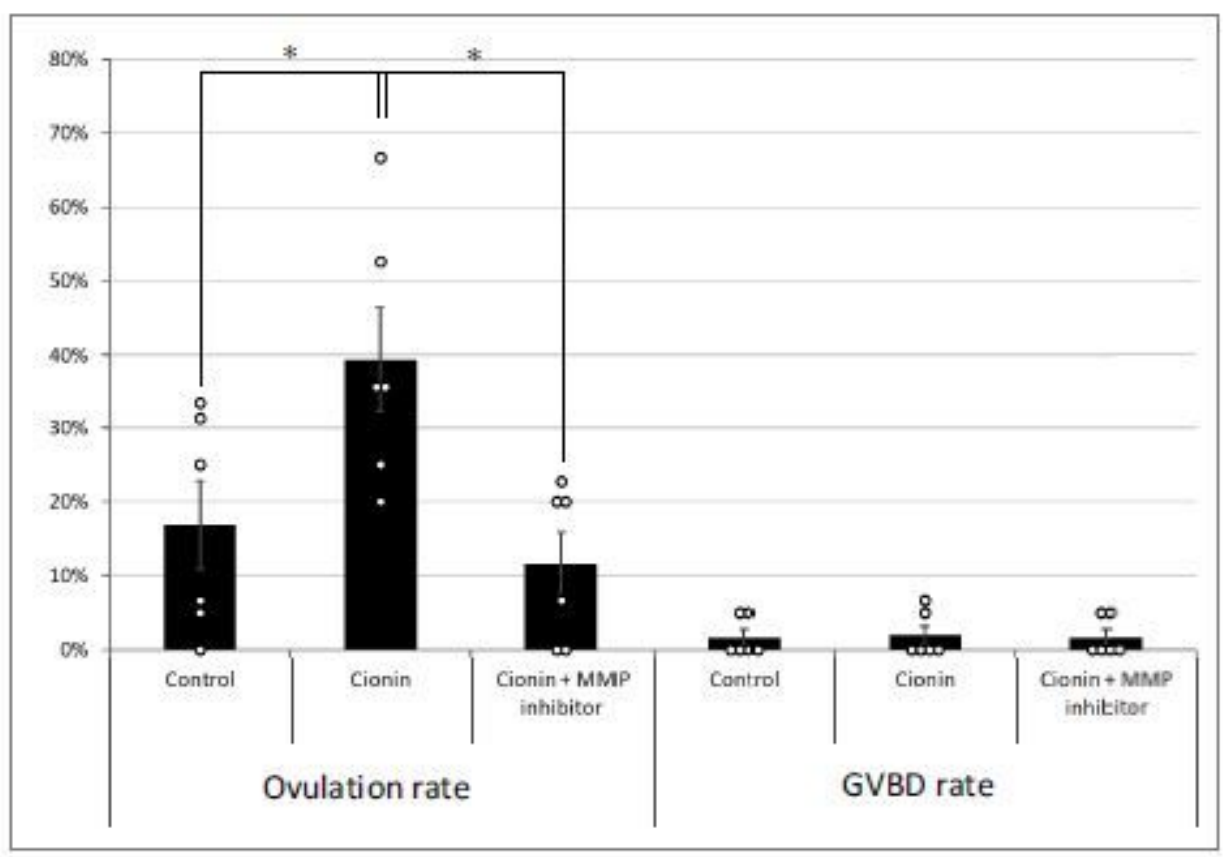

Figure 6

Effect of MMP2/9 inhibitor II on the ovulation and GVBD of cionin-treated follicles. (A) Representative images of follicles before and after treatment with $5 \mu \mathrm{M}$ cionin or $5 \mu \mathrm{M}$ cionin and $10 \mu \mathrm{M}$ MMP2/9 inhibitor II for $24 \mathrm{~h}$. Cionin-induced ovulation of stage II follicles was disrupted by MMP2/9 inhibitor II. (B) Ovulation and GVBD rates were calculated after the 24-h incubation. MMP2/9 inhibitor II significantly inhibited ovulation in the cionin-treated follicles. MMP2/9 inhibitor II did not affect GVBD rate. Results are means \pm SEM $(n=6)$. * $P<0.05$ control vs. cionin or cionin vs. MMP2/9 inhibitor II + cionin (one-way ANOVA followed by Tukey's post hoc test). 


\section{CiMmp2/9/13}

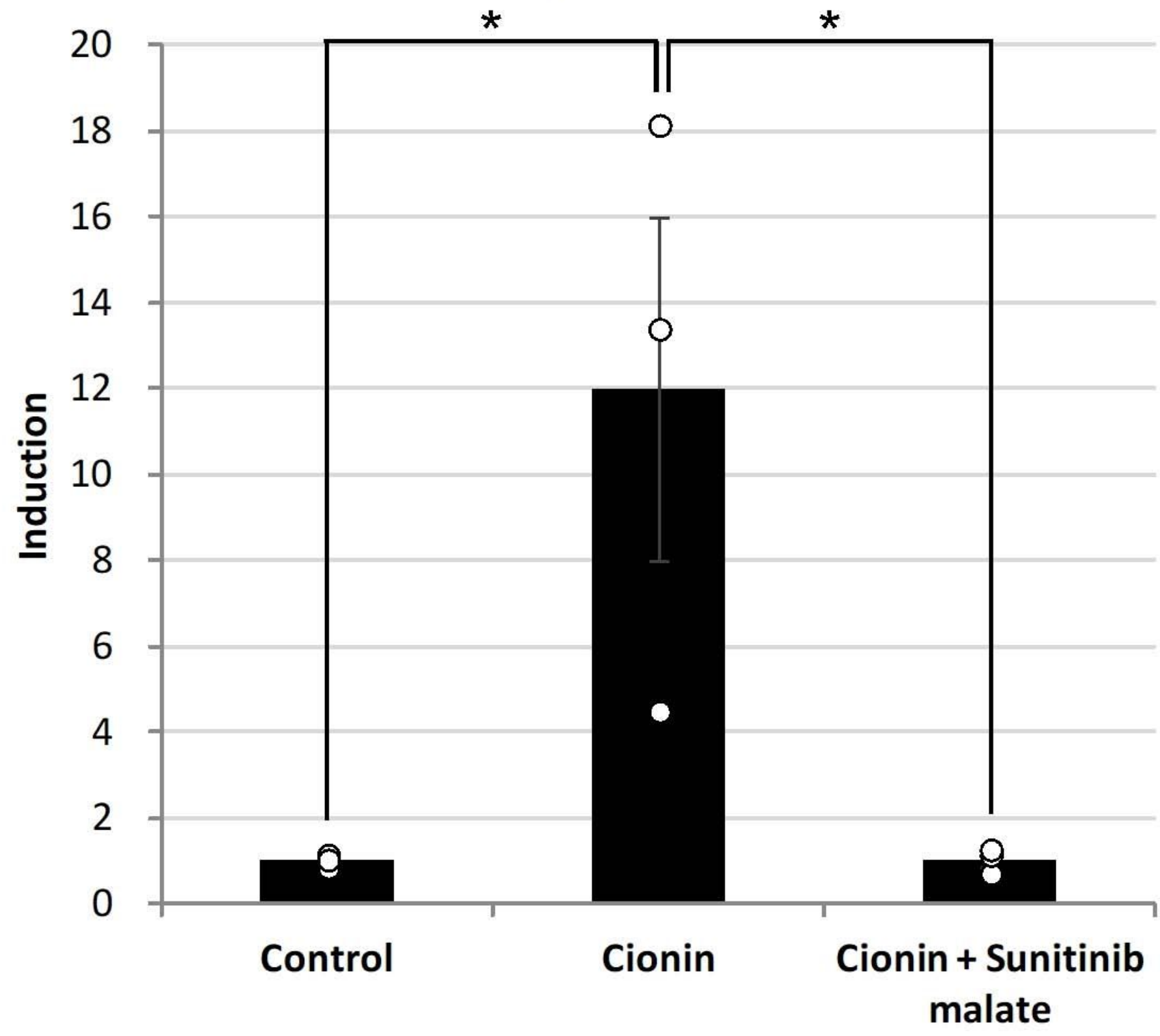

Figure 7

Real-time PCR analysis of cionin- and sunitinib malate-treated follicles. Sunitinib malate arrested the upregulation of the CiMmp2/9/13 gene by cionin after a 24-h incubation. Results are means \pm SEM ( $n=$ 3). * $\mathrm{P}<0.05$ control vs. cionin or cionin vs. cionin + sunitinib malate (one-way ANOVA followed by Tukey's post hoc test). 


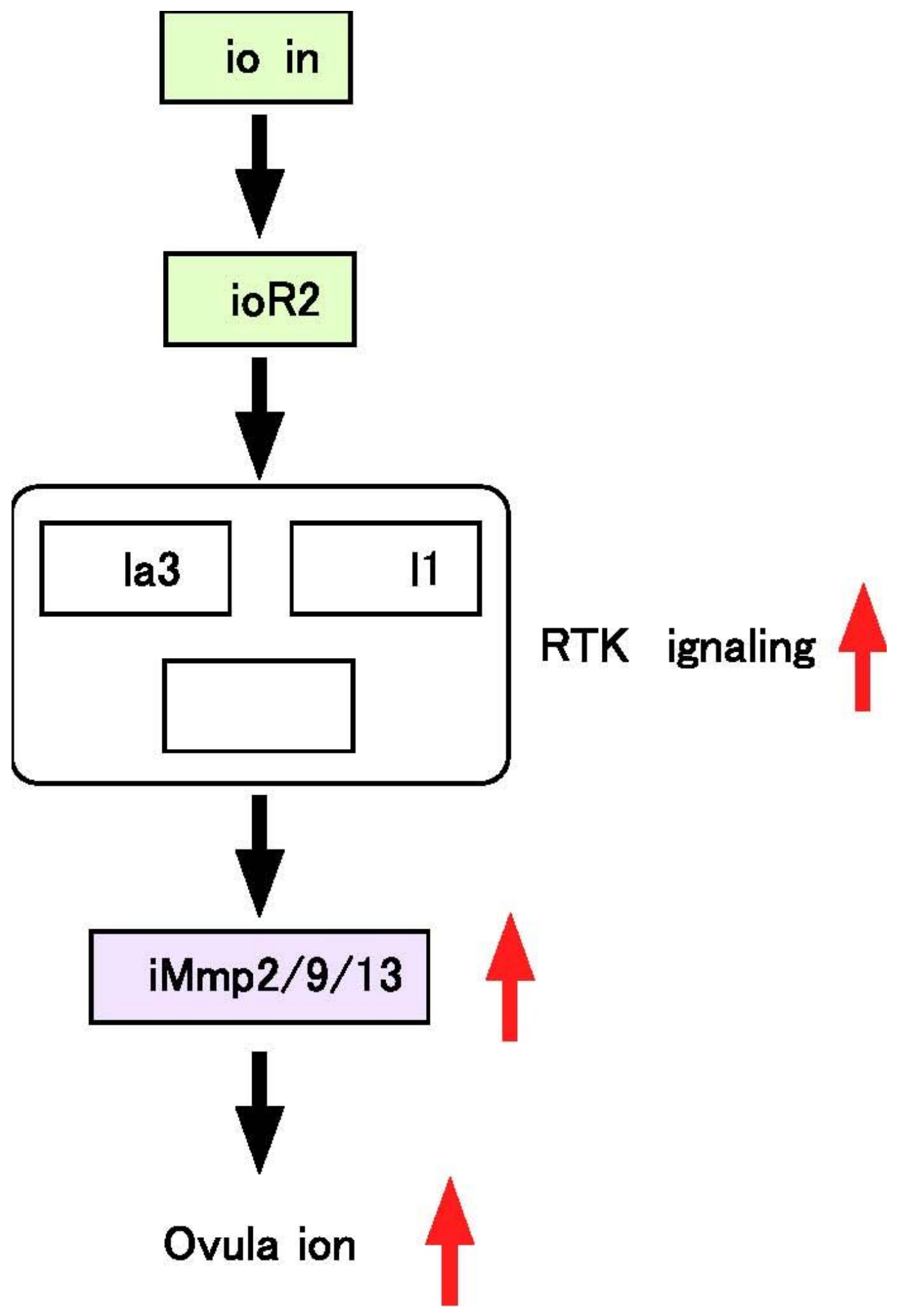

Figure 8

Schematic illustration of the regulatory mechanisms of cionin-directed ovulation. Cionin interacts with its receptor, CioR2, and activates RTK signaling. Subsequent induction of CiMmp2/9/13, which digests collagens in the outer follicular cell layer, leads to ovulation.

Supplementary Files 
This is a list of supplementary files associated with this preprint. Click to download.

- Osugietal2021cioninsupportinginformation.pdf

- Osugietal.2021SupplementalvideoforSciRep.mov

- Table1.jpg 